\title{
Tumores odontogénicos a células fantasmas. Conceptos actuales y aporte de 10 nuevos casos
}

\section{Odontogenic tumours ghost cell tumors. Actual concepts and reports of 10 new cases}

\author{
Keochgerián Basmayean V*, Legnani Moiana R**, Cosetti Olivera L***
}

\section{RESUMEN}

Se presentan 10 nuevos casos de neoplasias odontogénicas a células fantasmas del archivo de la Cátedra de Anatomía Patológica de la Facultad de Odontología de la UDELAR de Montevideo, Uruguay.

Estos tumores constituyeron el $0,08 \%$ del total de biopsias del Servicio y el 2,9\% de todas las neoplasias odontogénicas registradas. Nueve fueron tumores odontogénicos quísticos calcificantes y uno fue tumor dentinogénico a células fantasmas. Ocho casos fueron centrales y dos periféricos (uno, quístico y el otro, sólido).

Se registraron las características clínicas, radiográficas e histopatológicas y se compararon con otros trabajos de la literatura internacional.

Palabras clave: Tumores odontogénicos, tumores odontogénicos a células fantasmas, quiste odontogénico calcificante, tumor odontogénico quístico calcificante, tumor dentinogénico de células fantasmas.

\section{SUMMARY}

This paper reports 10 new cases of ghost cell odontogenic tumors retrieved from the files of the Oral Pathology Service of the Faculty of Dentistry (UDELAR), Montevideo, Uruguay. These tumors accounted for $0.08 \%$ of total biopsies and $2.9 \%$ of all registered odontogenic neoplasms. Nine cases were calcifying cystic odontogenic tumors (CCOT) and one dentinogenic ghost cell tumor (DGCT), eight in central and two in peripheral locations (one cystic and one solid variant).

Clinical, radiographic and histopathological features are presented and compared with reported cases in literature.

Key words: Odontogenic tumors, odontogenic ghost cells tumors; calcifying odontogenic cyst; calcifying cystic odontogenic tumors, dentinogenic ghost cell tumor.

Fecha de recepción: 20 de julio de 2011.

Aceptado para publicación: 10 de enero de 2012.

Keochgerián Basmayean V, Legnani Moiana R, Cosetti Olivera L. Tumores odontogénicos a células fantasmas. Conceptos actuales y aporte de 10 nuevos casos. Av. Odontoestomatol 2013; 29 (2): 63-72.

* Prof. Agregado de la Cátedra de Anatomía Patológica de la Fac. de Odontología UDELAR.

** Cirujano Bucomaxilofacial de SMI-IMPASA.

*** Prof. Adjunto de la Cátedra de Patología y Semiología y Clínica estomatológica UDELAR. Prof. Adjunto del Servicio de Urgencia de la Fac. de Odontología de la UDELAR. 


\section{INTRODUCCIÓN}

En el año 1962, Gorlin y col (1) describieron, por primera vez, una lesión morfológicamente quística con calcificaciones que fue denominada "quiste odontogénico calcificante". La presencia de células de tipo ameloblástico, la producción de material similar a dentina y la ocurrencia en sitios relacionados a piezas dentarias, determinaron su inclusión en el grupo de patologías odontogénicas.

En 1971 es reconocida como entidad por la OMS e incluida en el listado de neoplasias de origen odontogénico (2). Se señalaron sus características morfológicas principales: membrana quística epitelial con capa de células basales columnares bien definida, epitelio tipo retículo estelar de espesor variable, células epiteliales sin núcleo denominadas "fantasmas", a veces mineralizadas y material tipo dentina displásica relacionado con la membrana basal del quiste.

La comunicación de nuevos casos puso en evidencia la existencia de lesiones con similares características morfológicas, pero con estructura sólida. En 1981, Praetorious y col. (3) consideraron que deberían separarse dos tipos de lesiones a células fantasmas, una de naturaleza quística, el llamado "quiste odontogénico calcificante”, y otra neoplásica, que denominaron "tumor dentinogénico a células fantasmas". En la clasificación de quistes y tumores de los maxilares del año 1992, la OMS (4) adhiere al criterio dualista de Praetorious.

En el 2001, Yoshida (5) consideró que todas estas lesiones eran de naturaleza neoplásica, aún cuando la presentación macroscópica fuera quística (criterio monista).

En la última edición de 2005, lo expertos de la OMS (6) adoptan el criterio monista, denominando "Tumor Odontogénico Quístico Calcificante" (TOQC) a la forma quística y "Tumor Dentinogénico a Células Fantasmas" (TDCF) a la forma sólida. También se establece la existencia de la contraparte maligna, el "Carcinoma Odontogénico a Células Fantasmas" (COCF).

Clínicamente, pueden ser centrales o periféricos y, en ocasiones, se asocian con otros tumores odonto- génicos, principalmente odontomas compuestos o complejos y, en menor número, a ameloblastomas, fibroma ameloblástico, fibroodontoma ameloblástico, odontoameloblastoma y tumor odontogénico adenomatoide $(3,7)$.

Praetorious distinguió para las formas quísticas 4 subtipos que contemplan diferentes variantes morfológicas. El subtipo 1 corresponde a la forma quística simple; el 2 se asocia con odontoma complejo o compuesto, el subtipo 3 presenta proliferación ameloblastomatosa y el subtipo 4 se asocia a otros tumores odontogénicos, excluyendo a los odontomas y ameloblastomas.

Ledesma Montes y col. (8) publicaron recientemente un trabajo donde se estudiaron 122 casos de tumores a células fantasmas procedentes de 14 servicios de Anatomía Patológica Buco-Máxilo-Facial, de seis países diferentes. En este trabajo se aplica la sub división de Praetorious para las formas quísticas y se incluyen las formas sólidas (TDCF), las formas malignas (COCF) y las formas periféricas.

Frente a esta entidad tan controvertida, de presentación compleja y variada, que muchas veces resulta difícil catalogar, el propósito de esta publicación es aportar 10 nuevos casos del archivo de la Cátedra de Anatomía Patológica de la Facultad de Odontología de la Universidad de la República del Uruguay (UDELAR) y compararlos con los datos obtenidos en las series publicadas internacionalmente.

\section{MATERIALES Y MÉTODOS}

Se contabilizaron las biopsias recibidas desde el año 1960 al 2009. Se seleccionaron los casos de tumores odontogénicos centrales y periféricos, benignos y malignos, centrándose en los diferentes tipos de tumores odontogénicos a células fantasmas de acuerdo a los criterios actuales de la OMS.

Se consideraron los subtipos establecidos por Praetorious (3) y modificados por Ledesma Montes y col. (8). Se hizo la revisión histológica de las láminas originales de los tumores odontogénicos y, en algunos casos se realizaron nuevos cortes. Se tiñeron con HE y Tricrómico de Masson. 
Los casos de neoplasias a células Fantasmas fueron registrados en fichas donde se recabó la siguiente información: edad, género, raza, localización, tamaño, manifestaciones clínicas, registros imagenológicos, diagnóstico histopatológico definitivo, sub tipos y evolución (Tabla 1).

\section{RESULTADOS}

Se contabilizaron 12.069 biopsias, 344 fueron tumores odontogénicos de los cuales 10 fueron tumores de células fantasmas, 8 centrales y 2 periféricos.

Por lo tanto el $0,08 \%$ de las biopsias recibidas y el $2,85 \%$ de los tumores odontogénicos, fueron tumores de células fantasmas. Nueve casos correspondieron a tumor odontogénico quístico calcificante $(2,61 \%)$, de los cuales uno fue periférico; un caso $(0,24 \%)$ correspondió a tumor dentinogénico a células fantasmas de ubicación periférica. No se registraron casos de neoplasias malignas.

$80 \%$ de los casos fueron centrales y $20 \%$, periféricos.

El rango de edad en los 10 casos varió entre 12 y 76 años; el promedio fue de 51 y la mediana de 55 . Todos los casos ocurrieron en pacientes de raza blanca.

Siete casos fueron de género masculino y tres del femenino por lo cual el índice de masculinidad es de 2.33:1.

La mayoría se localizó en mandíbula (70\%) y los restantes en el maxilar superior (30\%).

Los tumores quísticos centrales mandibulares se situaron: uno en el sector anterior, dos en el sector lateral derecho, dos en el sector lateral izquierdo y otro ocupó todo el maxilar inferior.

En el maxilar superior uno se ubicó en el sector anterior y el otro en el sector anterolateral.

Ambas formas periféricas se encontraron en el sector anterior incisivo, uno mandibular y otro maxilar superior.

El tamaño varió desde 0,5 a $15 \mathrm{~cm}$. Las formas periféricas fueron las más pequeñas $(0,5$ y $1 \mathrm{~cm})$.

Tres casos deformaron ambas tablas, cuatro la tabla vestibular y uno el piso de fosas nasales.
Nueve fueron asintomáticos y uno (caso 2) presentó dolor.

La presentación radiográfica en cinco casos centrales fue radiolúcida unilocular bien delimitada. En los restantes casos uno mostró imagen radiolúcida multilocular bien definida con área radiopaca, otro, imagen radiolúcida unilocular bien delimitada con área radiopaca y un caso con imagen mixta sin límites claros.

Se asociaron a dientes retenidos en 4 casos (40\%) y reabsorciones radiculares de piezas erupcionadas en dos (25\%).

En ningún caso hubo correlación clínico-histopatológica. En 2 de ellos, el diagnóstico clínico presuntivo fue de quiste residual, odontoma quístico en un caso, quiste dentígero en otro, quiste odontogénico sin especificación en 2 casos y carcinoma en otro. Las lesiones periféricas se diagnosticaron como crecimiento sobre encía y quiste mandibular extraóseo.

Del punto de vista morfológico macroscópico nueve lesiones fueron quísticas y una sólida.

Cuatro casos centrales fueron quísticos simples (subtipo 1), dos se asociaron a odontoma complejo (subtipo 2) y dos con proliferación ameloblastomatosa (subtipo 3).

Todos presentaron células fantasmas en cantidad variable, en su mayoría sin núcleo o con modificaciones degenerativas y conservación de parte del material cromatínico. Muchas de ellas con calcificación distrófica. Con Tricrómico de Masson se evidencia la presencia de un material intensamente teñido de rojo dentro de estas células.

El tejido conjuntivo de todos los casos fue en su mayoría de tipo fibroso denso, con abundante vascularización y hemorragia intersticial. En los casos números 1-6 y 8 se observaron focos de cristales de colesterol y formaciones granulomatosas.

La evolución clínica fue favorable y sin recidivas en 8 de los casos. En dos casos centrales no se tienen datos de la evolución (Tabla 1).

Analizando las características de cada subtipo encontramos que los TOQC subtipo 1 constituyeron el $50 \%$. Los de subtipo 2 y el 3 constituyeron el $20 \%$ respectivamente y el único TDCF es el $10 \%$. Con 


\begin{tabular}{|c|c|c|c|c|c|c|c|c|c|}
\hline \multicolumn{10}{|c|}{ TABLA 1} \\
\hline Caso & $\begin{array}{l}\text { Edad } \\
\text { Género } \\
\text { Raza }\end{array}$ & $\begin{array}{l}\text { Locali- } \\
\text { zación }\end{array}$ & $\begin{array}{c}\text { Tamaño } \\
\text { (cm) }\end{array}$ & Clínica & Rx & $\begin{array}{l}\text { Diagnóstico } \\
\text { clínico }\end{array}$ & $\begin{array}{l}\text { Diagn. } \\
\text { HP y } \\
\text { subtipos }\end{array}$ & Otros & $\begin{array}{l}\text { Evolu- } \\
\text { ción }\end{array}$ \\
\hline 1 & $\begin{array}{c}12-M \\
B\end{array}$ & $\begin{array}{l}\text { MS. Zona } \\
\text { incisiva- } \\
\text { Central }\end{array}$ & $5 \mathrm{~cm}$ & $\begin{array}{l}\text { Crecimiento } \\
\text { lento } \\
\text { Piso fosas } \\
\text { nasales } \\
\text { elevado, } \\
\text { asintomático }\end{array}$ & $\begin{array}{l}\text { Unilocular } \\
\text { Radiolúcida } \\
\text { delimitada }\end{array}$ & Quiste & $\begin{array}{c}\text { TO.Q.C } \\
\text { tipo } 1\end{array}$ & $\begin{array}{c}11 \text { No } \\
\text { erupcionado }\end{array}$ & Sp \\
\hline 2 & $\begin{array}{c}32-M \\
B\end{array}$ & $\begin{array}{l}\text { MI. Zona } \\
\text { 32-36- } \\
\text { Central }\end{array}$ & $9 \mathrm{~cm}$ & $\begin{array}{l}\text { Dolor } \\
\text { punzante } \\
\text { zona } 36 \\
\text { deforma } \\
\text { tabla V y } \\
\text { reabsorción }\end{array}$ & $\begin{array}{l}\text { Unilocular } \\
\text { Radiolúcida } \\
\text { delimitada c/ } \\
\text { Área } \\
\text { radiopaca } \\
\text { anterior }\end{array}$ & $\begin{array}{l}\text { Odontoma } \\
\text { quístico }\end{array}$ & $\begin{array}{c}\text { TO Q.C } \\
\text { tipo } 2\end{array}$ & $\begin{array}{l}\text { Reabsorción } \\
33 \text { retenido }\end{array}$ & Sp \\
\hline 3 & $\begin{array}{c}76-M \\
B\end{array}$ & $\begin{array}{c}\text { MI. Zona } \\
\text { izquierda } \\
\text { Central }\end{array}$ & $4 \mathrm{~cm}$ & $\begin{array}{l}\text { Asintomático } \\
\text { Mucosa } \\
\text { ulcerada. } \\
\text { S/adenopatías. } \\
\text { Deformación } \\
\text { dos tablas }\end{array}$ & Mixta S/límites & Carcinoma & $\begin{array}{l}\text { TOQ.C } \\
\text { tipo } 3\end{array}$ & Desdentado & S/datos \\
\hline 4 & $\begin{array}{c}42-\mathrm{F} \\
\mathrm{B}\end{array}$ & $\begin{array}{l}\text { MI. 43-34 } \\
\text { Central }\end{array}$ & $7 \mathrm{~cm}$ & $\begin{array}{c}\text { Asintomático } \\
\text { Deformación } \\
\text { tabla V. }\end{array}$ & $\begin{array}{c}\text { Radiolúcida } \\
\text { unilocular } \\
\text { delimitada }\end{array}$ & $\begin{array}{c}\text { Quiste } \\
\text { odontogénico }\end{array}$ & $\begin{array}{c}\text { T O Q C } \\
\text { tipo } 1\end{array}$ & $\begin{array}{c}43 \text { No } \\
\text { erupcionado }\end{array}$ & Sp \\
\hline 5 & $\begin{array}{c}55-M \\
B\end{array}$ & $\begin{array}{l}\text { MI. Zona } \\
46 \\
\text { Central }\end{array}$ & $2,5 \mathrm{~cm}$ & $\begin{array}{c}\text { Drenaje } \\
\text { purulento } \\
\text { Deformación } \\
\text { tabla V }\end{array}$ & $\begin{array}{l}\text { Radiolúcido } \\
\text { unilocular } \\
\text { delimitado }\end{array}$ & $\begin{array}{l}\text { Quiste } \\
\text { residual }\end{array}$ & $\begin{array}{c}\text { T O Q C } \\
\text { tipo } 1\end{array}$ & $\begin{array}{c}\text { Antecedentes } \\
\text { de cirugía } \\
\text { de quiste od. } \\
\text { y extr. del } 46\end{array}$ & Sp \\
\hline 6 & $\begin{array}{c}34-M \\
B\end{array}$ & $\begin{array}{l}\text { MI. 48-37 } \\
\text { Central }\end{array}$ & $15 \mathrm{~cm}$ & $\begin{array}{c}\text { Asintomático } \\
\text { Deformación } \\
\text { dos tablas }\end{array}$ & $\begin{array}{c}\text { Radiolúcida } \\
\text { multilocular } \\
\text { c/radiopacidad } \\
\text { anterior }\end{array}$ & $\begin{array}{c}\text { Quiste } \\
\text { dentígero }\end{array}$ & $\begin{array}{l}\text { TOQC } \\
\text { tipo } 2\end{array}$ & $\begin{array}{l}43 \text { y } 32 \text { no } \\
\text { erupcionados } \\
\text { Reabsorción } \\
\text { raíces infer. }\end{array}$ & Sp \\
\hline 7 & $\begin{array}{c}76-M \\
B\end{array}$ & $\begin{array}{l}\text { MS. Zona } \\
22-25 \\
\text { Central }\end{array}$ & $4 \mathrm{~cm}$ & $\begin{array}{c}\text { Asintomático } \\
\text { Deformación } \\
\text { de tabla V }\end{array}$ & $\begin{array}{l}\text { Unilocular } \\
\text { Radiolúcida } \\
\text { delimitada }\end{array}$ & $\begin{array}{l}\text { Quiste } \\
\text { residual } \\
\text { o fisurario }\end{array}$ & $\begin{array}{l}\text { TOQC } \\
\text { tipo } 3\end{array}$ & $\begin{array}{l}\text { Zona } \\
\text { desdentada } \\
\text { Punción de } \\
\text { líquido } \\
\text { quístico }\end{array}$ & Sp \\
\hline 8 & $\begin{array}{c}61-\mathrm{F} \\
\mathrm{B} \\
\text { Central }\end{array}$ & $\begin{array}{l}\text { MI. Zona } \\
\text { del 43-45 }\end{array}$ & $\begin{array}{c}2 \mathrm{~cm} \\
\text { dos tablas }\end{array}$ & $\begin{array}{c}\text { Asintomática } \\
\text { Deformación } \\
\text { bien delimiada }\end{array}$ & $\begin{array}{c}\text { Radiolúcida } \\
\text { unilocular }\end{array}$ & $\begin{array}{l}\text { Quiste } \\
\text { residual }\end{array}$ & $\begin{array}{l}\text { T OQC } \\
\text { tipo } 1 \\
\text { citrino }\end{array}$ & $\begin{array}{l}\text { Punción de } \\
\text { líquido }\end{array}$ & S/datos \\
\hline 9 & $\begin{array}{c}56-F \\
B\end{array}$ & $\begin{array}{c}\text { Zona } \\
\text { incisiva } \\
\text { Palatina }\end{array}$ & $0,5 \mathrm{~cm}$ & $\begin{array}{l}\text { Crecimiento } \\
\text { en encía. } \\
\text { Sesil, lisa } \\
\text { blanda } \\
\text { Asintomática }\end{array}$ & - & $\begin{array}{l}\text { Crecimiento } \\
\text { sobre encía }\end{array}$ & $\begin{array}{c}\text { T.DCF } \\
\text { periférico }\end{array}$ & - & Sp \\
\hline 10 & $\begin{array}{c}60-M \\
B\end{array}$ & $\begin{array}{c}\text { Mucosa V. } \\
32-33\end{array}$ & $1 \mathrm{~cm}$ & $\begin{array}{l}\text { Lesión } \\
\text { elevada } \\
\text { sesil lisa }\end{array}$ & - & $\begin{array}{c}\text { Quiste } \\
\text { extraóseo } \\
\text { mandibular }\end{array}$ & $\begin{array}{c}\text { T.OQ.C } \\
\text { periférico }\end{array}$ & - & Sp \\
\hline
\end{tabular}


respecto al tamaño de las lesiones centrales, las del subtipo 1 tienen un promedio de longitud de $4,1 \mathrm{~cm}$, las del subtipo $2,12 \mathrm{~cm}$, el subtipo $3,4 \mathrm{~cm}$ y los tumores periféricos $0,75 \mathrm{~cm}$ (Tabla 2).

La distribución por edad de las neoplasias centrales es variable. Los TOQC, subtipo 1 , tienen un rango de edades de 12 a 61 años con promedio de 42,5. Las formas de subtipo 2 se dan en la $4^{a}$ década, con promedio 33 años. En el subtipo 3, los dos casos fueron en pacientes de la sexta década (Tabla 2).

\section{TABLA 2}

\begin{tabular}{|l|c|c|c|}
\hline $\begin{array}{l}\text { Tipos } \\
\text { y subtipos }\end{array}$ & $\begin{array}{c}\text { Rango } \\
\text { de edad }\end{array}$ & Década & $\begin{array}{c}\text { Tamaño } \\
\text { Promedio }\end{array}$ \\
\hline TOQC subtipo1 & $12-55$ & $2^{\mathrm{a}}-6^{\mathrm{a}}$ & $4,10 \mathrm{~cm}$ \\
TOQC subtipo2 & $32-34$ & $4^{\mathrm{a}}$ & $12,00 \mathrm{~cm}$ \\
TOQC subtipo3 & 76 & $8^{\mathrm{a}}$ & $4,00 \mathrm{~cm}$ \\
Formas periféricas & $56-60$ & $6^{\mathrm{a}}$ & $0,75 \mathrm{~cm}$ \\
\hline
\end{tabular}

En lo referente al género, los casos del subtipo 1 se dan por igual en varones que en mujeres; en los subtipos 2 y 3 son en varones.

\section{DISCUSIÓN}

Los tumores a células fantasmas son lesiones odontogénicas de poca frecuencia.

En la presente casuística constituyen el 0,08\% de las biopsias recibidas y $2,9 \%$ de todos los tumores odontogénicos.

Las formas quísticas benignas (TOQC) son las más comunes. La OMS las define como neoplasias quísticas de origen odontogénico con epitelio tipo ameloblástico y células fantasmas que se pueden calcificar (6). Los tumores sólidos centrales, los periféricos y los malignos son de hallazgo raro $(9,10,11)$. Las formas sólidas se denominan Tumor Dentinogénico a Células Fantasmas (TDCF) y se definen como "neoplasia odontogénica localmente invasora, caracterizada por presentar islas de epitelio ameloblástico en estroma conjuntivo maduro". Se puede encontrar queratiniza- ción aberrante en forma de células fantasmas asociadas a variable cantidad de dentina displásica (6). Los estudios de inmunohistoquímica realizados por Yoshida (5) establecen que la inmunorreactividad de ambas lesiones es similar, por lo cual tanto las formas sólidas como las quísticas deben considerarse neoplasias (criterio monista).

En los 10 casos de tumores odontogénicos a células fantasmas examinados aquí, no se halló ningún tumor dentinogénico a células fantasmas central.

La contraparte maligna se conoce con diferentes denominaciones: Carcinoma odontogénico calcificante a células fantasmas, tumor odontogénico epitelial maligno a células fantasmas o tumor odontogénico epitelial agresivo a células fantasmas. Para la OMS estas neoplasias muestran hallazgos de tumor odontogénico quístico calcificante y/o de tumor dentinogénico a Células Fantasmas con actividad mitótica importante, atipia nuclear y pleomorfismo celular (6). Estas formas malignas son raras. Para Ledesma Montes y col. (8) constituyen el 2,46\%. En la casuística de la UDELAR no hubo casos.

También las neoplasias a células fantasmas periféricas son raras. En el estudio de Nagao no se registró ningún caso (12). Para este autor esto se explica por el frecuente error diagnóstico de catalogarlas como ameloblastomas u otros tumores odontogénicos periféricos. Aunque constituyen lesiones raras, en diferentes trabajos se han registrado variable número de casos (13). En el presente constituyen el $20 \%$ del total de casos registrados, se presentan en adultos, en la sexta década de la vida y en el sector anterior de los maxilares (Figuras 1, 2 y 3). Kumar y col. (14) analizaron los casos publicados en la literatura internacional, concluyendo que son más comunes en adultos mayores y con frecuente asiento en zona canino-premolar; estos datos coinciden con nuestros dos casos.

Del análisis de los resultados aquí obtenidos, los tumores odontogénicos quísticos calcificantes son el $80 \%$ mientras que para Ledesma Montes y col. son el 92,6\%. Considerando cada subtipo histológico, las formas quísticas simples, subtipo 1 , son el $50 \%$; para Ledesma Montes el 64,76\%. En segundo lugar se ubican las formas asociadas a odontomas (subtipo 2) que aquí son el 20\%; 22,13\% para Ledesma Mon- 


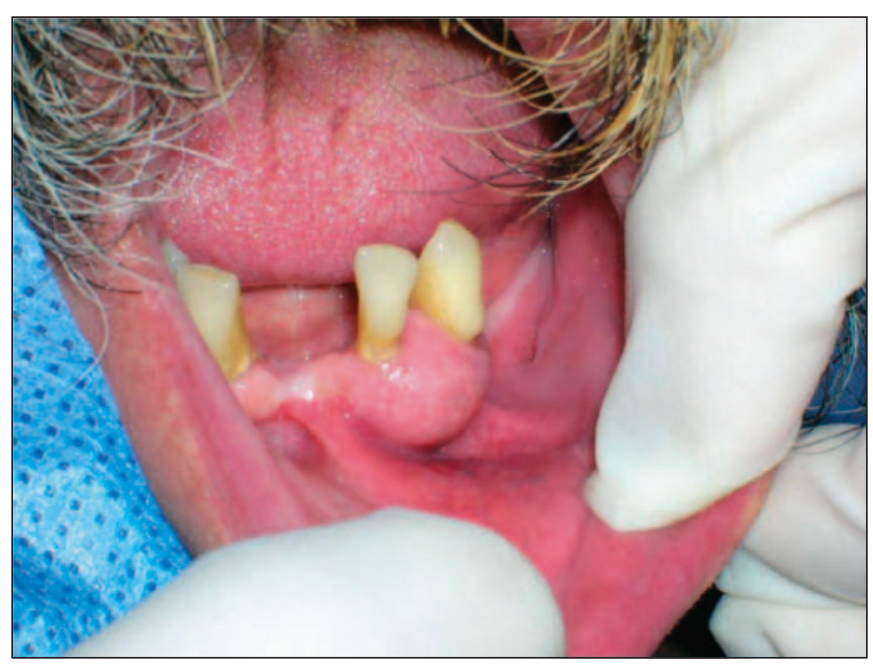

Fig. 1. Caso $n^{\circ}$ 10. Tumoración gingival a nivel del 32 y 33. Color de la encía rosado, graneado.

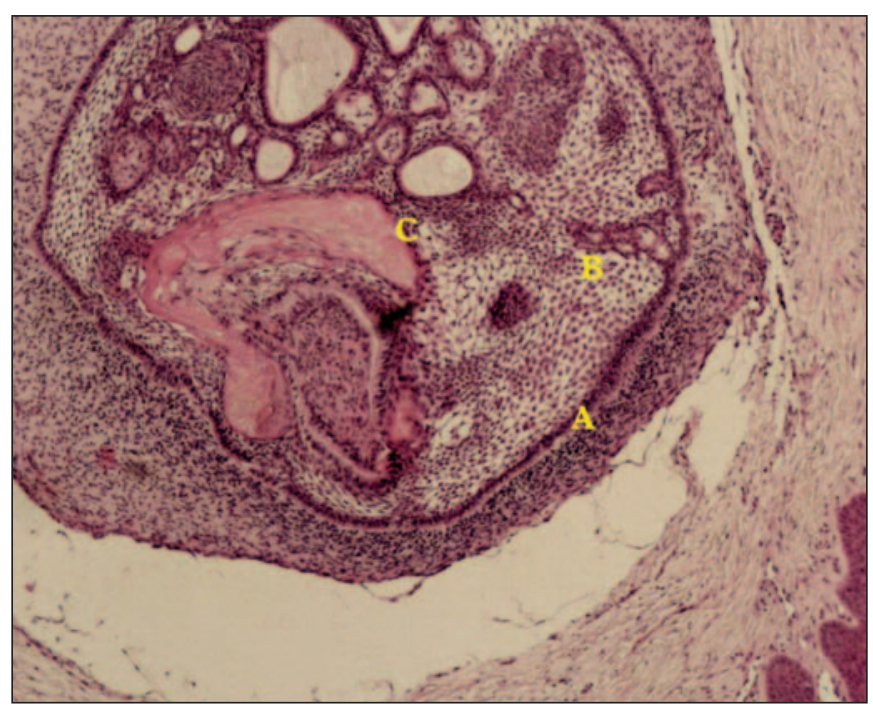

Fig 2. Caso $n^{\circ}$ 9. Tumor dentinogénico a células fantasmas periférico. Microscopía HE 40x. Crecimiento neoplásico subgingival. Lesión circunscrita, sólida. A: Epitelio ameloblástico. B: Tejido tipo retículo estelar. C: Células fantasmas.

tes y col., y 22 a $47 \%$ para Reichart y col (15). Existe acuerdo general que la mayoría de los casos se asocian a odontoma compuesto y raramente a odontoma complejo (15). Los dos casos de esta serie correspondieron a odontomas de tipo complejo.

Mucho se ha discutido acerca del mecanismo patogénico que determina la coexistencia de un odontoma con el tumor odontogénico quístico calcificante.
Hay autores que consideran que el odontoma se produce por inducción del epitelio del tumor quístico sobre el conectivo (16). Sin embargo la hipótesis más aceptada (15) es que un odontoma evolucionaría a una forma quística donde su componente epitelial induce la formación de la lesión a células fantasmas. Un hecho llamativo que apoya esta última interpretación es que 18 \% de los odontomas contienen células fantasmas (15).

Del subtipo 3, se registraron dos casos, constituyendo el $20 \%$ restante. Para el estudio colaborativo es el $2,46 \%$. Las formas sólidas son aquí el $10 \%$ y para Ledesma Montes 4,92\%. Estas diferencias posiblemente estén vinculadas al bajo número de casos de la presente casuística.

Considerando el grupo etario más afectado surge, al igual que en otros trabajos $(1,3,7,13)$ que estas lesiones se dan en un amplio rango de edades, de 12 a 76 años, con una mediana de 55 años y una media de 51. Excepcionalmente se presentan en la infancia; Oliveira y col. (17) comunicaron un caso en un niño de tres años que correspondió al subtipo 2 (asociado a odontoma). Para Hong estas formas son más frecuentes en la segunda década con un promedio de 14,7 años (13). Los dos casos asociados a odontoma de la presente serie correspondieron a pacientes de la tercera y cuarta década.

Se ha señalado que no existe predilección por género ni raza $(1,3,7,13)$. Aquí existió leve predominio masculino y todos fueron de raza blanca, condición que podría explicarse por las características raciales del Uruguay (Tabla 1).

En cuanto a la localización, la mayoría de los autores señalan que se presentan indistintamente en ambos maxilares. En el presente trabajo son más frecuentes en la mandíbula al igual que en las series de Hong y de Praetorious $(13,3)$. En cambio otros autores encuentran mayor número en el maxilar superior (17).

El tamaño de la lesión también es variable, la mayoría no pasa los $4 \mathrm{~cm}$ y raramente sobrepasan los $8 \mathrm{~cm}$ (7). En la serie de Li y Yu (18) sólo cuatro de tipo 1 excedieron los $2 \mathrm{~cm}$ y dos de tipo 2 alcanzaron $13 \mathrm{~cm}$ después de dos recidivas. Aquí se presenta un caso que alcanzó "de novo" $15 \mathrm{~cm}$ de eje mayor e involu- 

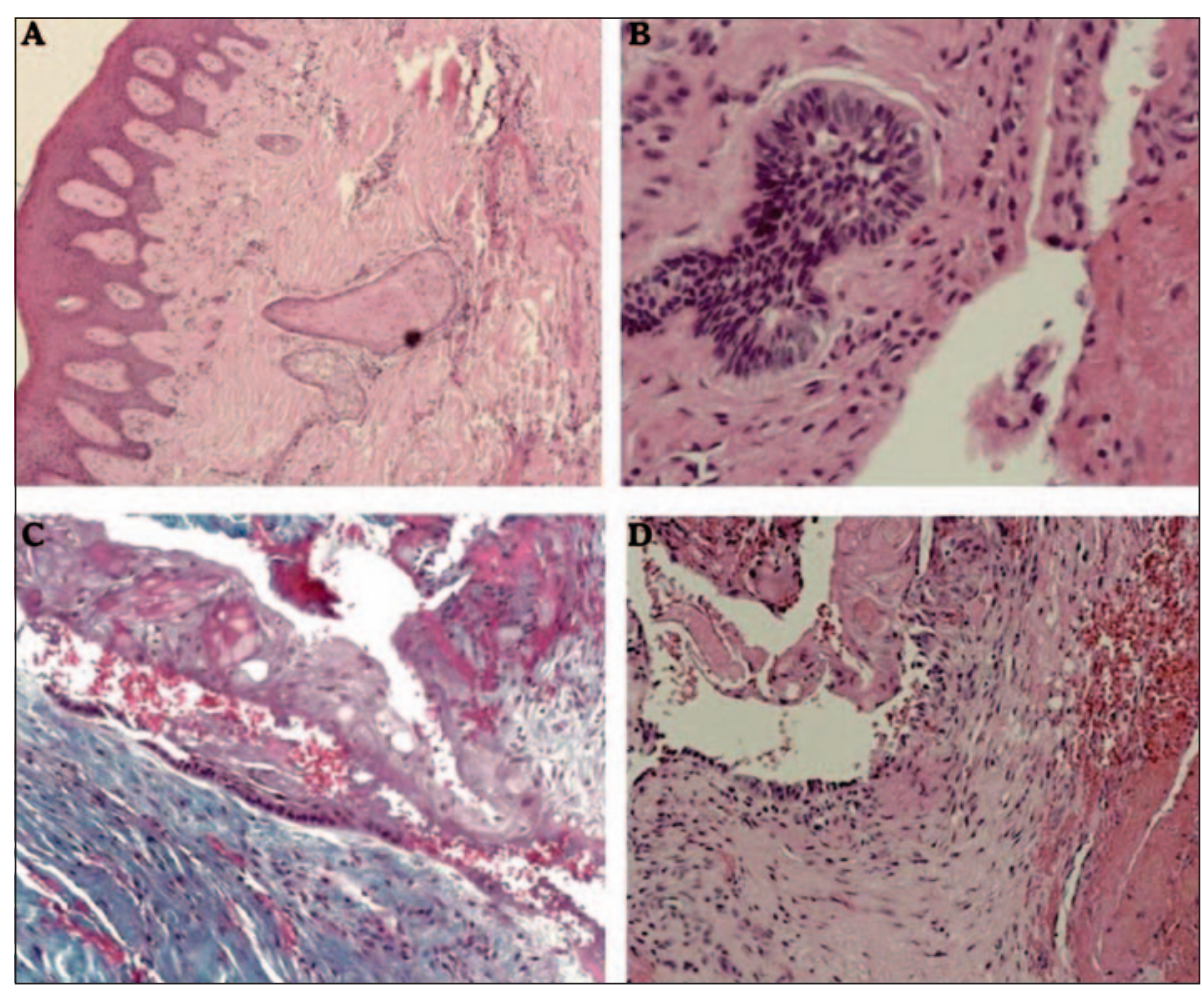

Fig. 3. Caso $n^{\circ}$ 10. Tumor odontogénico quístico calcificante periférico. A: 40x HE, epitelio de cubierta. Islotes de epitelio odontogénico activo en la lámina propia gingival. B: 100x HE, epitelio odontogénico activo. C: $100 x$ tricrómico de Masson, tumor odontogénico quístico. Se aprecia el epitelio delgado tipo ameloblástico; en la luz células fantasmas. Cápsula conjuntiva fibrosa con escaso exudado inflamatorio. D: 200x HE, epitelio delgado. Células fantasmas en la luz quística. cró a todo el maxilar inferior (Figura 4). Con tratamiento quirúrgico conservador evolucionó favorablemente y se ha mantenido sin lesión hasta el presente.

El desplazamiento asintomático de una o dos tablas es común a todas las formas centrales, coincidiendo con lo encontrado en otras casuísticas (15) (Figura 5).

Los hallazgos radiográficos son variados: radiolúcidos uni o multilocular, de límites bien definidos, con material calcificado radio-opaco en variadas cantidades o grandes masas radio-opacas cuando se asocian con odontoma. Otros hallazgos radiográficos son la reabsorción radicular, presente en $25 \%$ de los casos y los desplazamientos dentarios. Se ha establecido que el $32 \%$ de los casos presentan dientes no erupcionados (5). En esta casuística son el 37,5\%.

Entre los hallazgos histopatológicos de estas lesiones, las células fantasmas constituyen un elemento morfológico patognomónico; están en el 100\% de

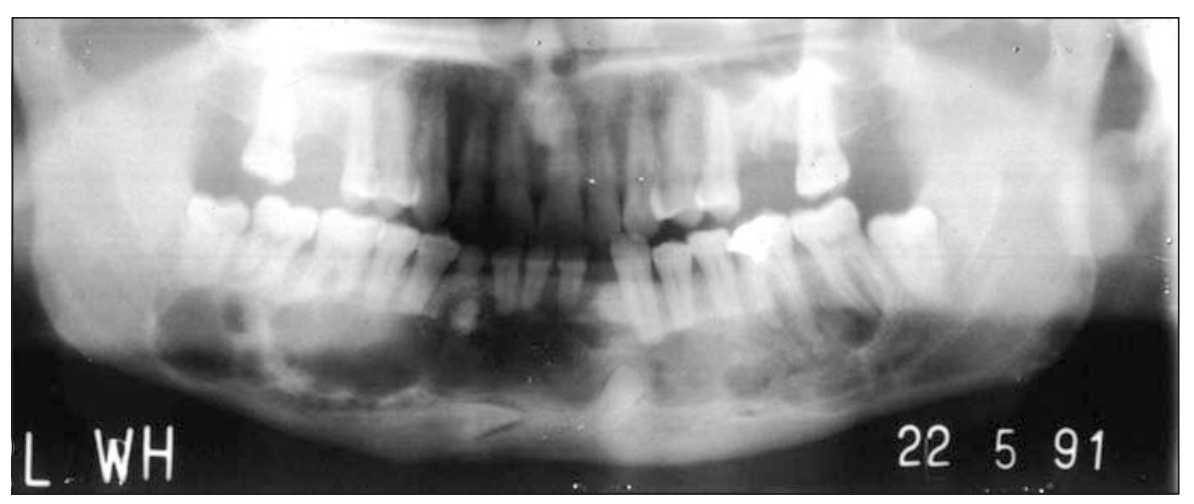

Fig. 4. Caso $n^{\circ}$ 6. Opt. Obsérvese área radiolúcida multilocular extendida del 37 al 48. Dientes 43 y 32 retenidos cerca de la basal en el sector anterior. Área radiopaca (odontoma) debajo del 83. Reabsorción de raíz del 46, 45, 44, 33, 34, 35. 

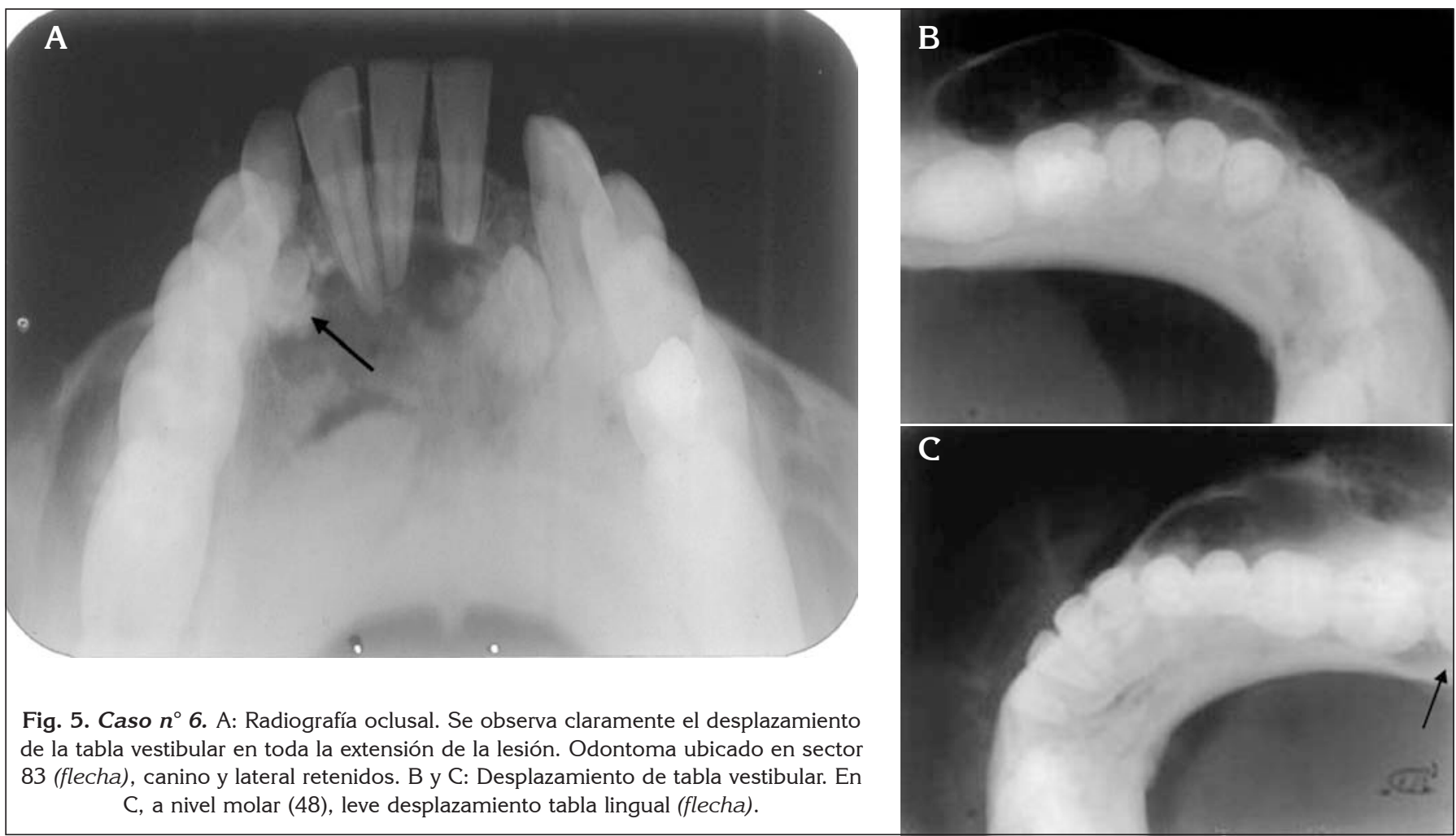

Fig. 5. Caso $n^{\circ}$ 6. A: Radiografía oclusal. Se observa claramente el desplazamiento de la tabla vestibular en toda la extensión de la lesión. Odontoma ubicado en sector 83 (flecha), canino y lateral retenidos. B y C: Desplazamiento de tabla vestibular. En C, a nivel molar (48), leve desplazamiento tabla lingual (flecha).

los casos. Cabe consignar que estas células también se pueden presentar en otras lesiones odontogénicas (odontomas, ameloblastomas, fibroma ameloblástico), aunque no como hallazgo constante. Son células grandes, eosinófilas, pálidas que han perdido su núcleo y fueron interpretadas inicialmente, como epiteliales queratinizadas (1). Estudios recientes con inmunohistoquímica revelan positividad para la amelogenina y otras proteínas del esmalte $(5,19)$. Por lo tanto, constituirían células odontogénicas epiteliales con diferenciación ameloblástica que producen material tipo matriz de esmalte acumulado en su citoplasma durante el proceso patológico. En trabajos experimentales aplicando técnicas de inmunohistoquímica durante la organogénesis dentaria, se demostró que la amelogenina es segregada en etapas tardías de la diferenciación de los preameloblastos y en etapas iniciales de diferenciación de los ameloblastos secretores (20). En estadios iniciales de la amelogénesis el esmalte en desarrollo se tiñe de rojo intenso con Tricrómico de Masson. Esta reactividad es similar a la observada en las células fantasmas (Figura 6), por lo cual se reafirma la hipóte- sis de que éstas serían células odontogénicas con diferenciación ameloblástica.

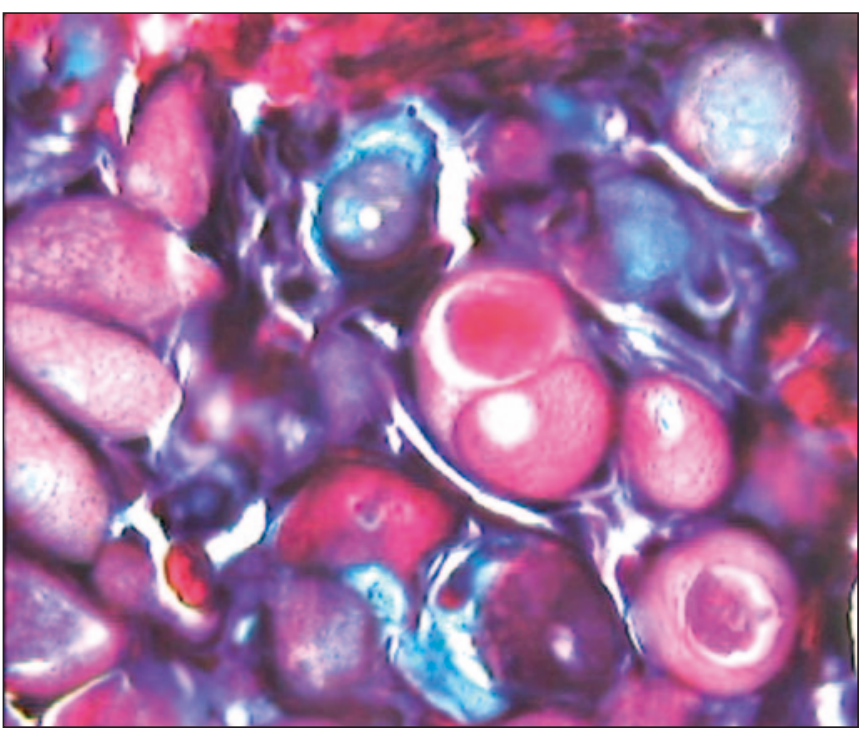

Fig. 6. 400x tricrómico de Masson. Células fantasmas. Material teñido de rojo intracelular interpretado como amelogenina. 
En conclusión se presentan diez casos de una entidad que, a pesar de haber sido descrita hace muchos años, sigue siendo discutida. Del análisis de los resultados no surgen grandes diferencias con otras casuísticas publicadas. Las formas quísticas simples son las más comunes; su localización más frecuente es en el maxilar inferior; se dan en amplio rango de edad; el tamaño es variable siendo las formas periféricas las más pequeñas. La aplicación de técnicas de inmunohistoquímica y los estudios con microscopio electrónico han permitido avanzar sobre el conocimiento de su naturaleza. A pesar de ello, su denominación, etiopatogenia y comportamiento clínico, deberán clarificarse mejor. Para ello es fundamental la comunicación de nuevos casos.

\section{BIBLIOGRAFÍA}

1. Gorlin RJ, Pindborg JJ, Clausen FP, Vickers RA. The calcifying odontogenic cyst - a possible analogue of cutaneous calcifying epithelioma of Malherbe: an analysis of fifteen cases. Oral Surg Oral Med Oral Pathol 1962;15:1235-43.

2. Pindborg JJ, Kramer IRH, Torconi H. Histological typing of odontogenic tumours, jaw cysts and allied lesions. Geneva: WHO; 1971. (WHO International histological classification of tumours).

3. Praetorius F, Hjorting-Hansen E, Gorlin RJ, Vickers RA. Calcifying odontogenic cyst. Range, variations and neoplastic potencial. Acta Odontol Scand 1981;39:227-40.

4. Kramer IRH, Pindborg JJ, Shear M. Histological typing of odontogenic tumours. Berlin: SpringerVerlag; 1992. (WHO International histological classification of tumours).

5. Yoshida M, Kumamoto H, Ooya K, Mayanagi $\mathrm{H}$. Histopathological and immunohistochemical analysis of calcifying odontogenic cysts. J Oral Pathol Med 2001;30:582-5.

6. Barnes L, Eveson JW, Reichart P, Sidransky D, editors. Pathology and genetics of head and neck tumours. $3^{\text {rd }}$ ed. Lyon: IARC; 2005. (WHO Classification of tumors, vol. 9).
7. Buchner A. The central (intraosseous) calcifying odontogenic cyst: an analysis of 215 cases. J Oral Maxillofac Surg 1991;49:330-9.

8. Ledesma-Montes C, Gorlin RJ, Shear M, Praetorious F, Mosqueda-Taylor A, Altini $M$ et al. International collaborative study on ghost cell odontogenic tumors: calcifying cystic odontogenic tumour, dentinogenic ghost cell tumour and ghost cell odontogenic carcinoma. J Oral Pathol Med 2008;37:302-8.

9. Orsini G, Fioroni M, Rubini C, Piattelli A. Periferal calcifying odontogenic cyst. J Clin Periodontol 2002;29:83-6.

10. Buchner A, Merrell PW, Hansen LS, Leider AS. Peripheral (extraosseous) calcifying odontogenic cyst: a review of forty-five cases. Oral Surg Oral Med Oral Pathol. 1991;72:65-70.

11. Lu Y, Mock D, Takata T, Jordan RC : Odontogenic ghost cell carcinoma: report of four new cases and review of the literature. J Oral Pathol Med 1999; 28:323-9.

12. Nagao T, Nakajima T, Fukushima M, Ishiki T. Calcifying odontogenic cyst : a survey of 23 cases in the Japanese literature. J Maxillofac Surg 1983; 11:174-9.

13. Hong SP, Ellis GL, Hartman KS. Calcifying odontogenic cyst. A review of ninety- two cases with reevaluation of their nature as cysts or neoplasms, the nature of ghost cells, and subclassification. Oral Surg Oral Med Oral Pathol 1991; 72:56-64.

14. Kumar U, Vij H, Kharbanda J, Aparna I, Radhakrishnan R. Dentinogenic ghost cell tumor of the peripherals variant mimicking epulis. Int $\mathrm{J}$ Dent [revista online] 2010 [citado 30 de mayo de 2011]; 2010:519494. Epub 2010 Oct 26. http:// www.ncbi.nlm.nih.gov/pmc/articles/PMC2964905/ ?tool=pubmed.

15. Reichart PA, Philipsen HP. Odontogenic tumours and allied lesions. London: Quintessence; 2004. 
16. Toida M. So called calcifying odontogenic cyst: review and discussion on the terminology and classification. J Oral Pathol Med 1998;27:49-52.

17. Oliveira JA, da Silva CJ, Costa IM, Loyola AM. Calcifying odontogenic cyst in infancy: report of case associated with compound odontoma. ASDC J Dent Child 1995;62:70-3.

18. Li TJ, Yu SF. Clinicopathologic spectrum of the so-called calcifying odontogenic cysts: a study of 21 intraosseous cases with reconsideration of the terminology and classification. Am J Surg Pathol 2003;27:372-84.

19. Takata T, Zhao M, Nikai H, Uchida T, Wang T. Ghost cells in calcifying odontogenic cyst express enamel-related proteins. Histochem J 2000;32: 223-9.

20. Uchida T, Tanabe T, Fukae M. Immunocytochemical localization of amelogenins in the deciduous tooth germs of the human fetus. Arch Histol Cytol 1989;52:543-52.

\section{CORRESPONDENCIA}

Verónica Keochgerián

Con.Castro129bis

Montevideo Rou

E-mail: verokeoc@hotmail.com 